EPJ Web of Conferences 64, 05004 (2014)

DOI: $10.1051 /$ epjconf/ 20146405004

(C) Owned by the authors, published by EDP Sciences, 2014

\title{
Role of local absorption on the X-ray emission from MHD accre- tion shocks in classical T Tauri stars
}

\author{
Bonito $^{1,2, a}$, Orlando ${ }^{2}$, Argiroffi $^{1,2}$, Miceli $^{2}$, Reale $^{1,2}$, Peres $^{1,2}$, Matsakos $^{3,4,5}$, Stehle $^{5}$, and lbgui ${ }^{5}$ \\ ${ }^{1}$ Dipartimento di Fisica e Chimica, Universita' degli Studi di Palermo, Piazza del Parlamento 1, 90134 \\ Palermo, Italy \\ ${ }^{2}$ INAF - Osservatorio Astronomico di Palermo, Piazza del Parlamento 1, 90134 Palermo, Italy \\ ${ }^{3}$ CEA, IRAMIS, Service Photons, Atomes et Molecules, 91191 Gif-sur-Yvette, France \\ ${ }^{4}$ Laboratoire AIM, CEA/DSM - CNRS - Universite' Paris Diderot, IRFU/Service d'Astrophysique, CEA Saclay, \\ Orme des Merisiers, 91191 Gif-sur-Yvette, France \\ ${ }^{5}$ LERMA, Observatoire de Paris, Universite' Pierre et Marie Curie and CNRS, 5 Place J. Janssen, 92195 \\ Meudon, France
}

\begin{abstract}
Accretion processes onto classical T Tauri stars (CTTSs) are believed to generate shocks at the stellar surface due to the impact of supersonic downflowing plasma. Although current models of accretion streams provide a plausible global picture of this process, several aspects are still unclear. For example, the observed X-ray luminosity in accretion shocks is, in general, well below the predicted value. A possible explanation discussed in the literature is in terms of significant absorption of the emission due to the thick surrounding medium. Here we consider a 2D MHD model describing an accretion stream propagating through the atmosphere of a CTTS and impacting onto its chromosphere. The model includes all the relevant physics, namely the gravity, the thermal conduction, and the radiative cooling, and a realistic description of the unperturbed stellar atmosphere (from the chromosphere to the corona). From the model results, we synthesize the X-ray emission emerging from the hot slab produced by the accretion shock, exploring different configurations and strengths of the stellar magnetic field. The synthesis includes the local absorption by the thick surrounding medium and the Doppler shift of lines due to the component of plasma velocity along the line-of-sight. We explore the effects of absorption on the emerging X-ray spectrum, considering different inclinations of the accretion stream with respect to the observer. Finally we compare our results with the observations.
\end{abstract}

\section{Introduction}

The study of the properties of the streams accreting material on a classical T Tauri star (CTTS) is interesting in a wide context, from the evolution of the young star itself, to the dynamics of the disk surrounding the star, to the possible formation of a planetary system. Well established accretion theories suggest that the plasma accreting on CTTSs and falling at approximately the free-fall velocity

ae-mail: sbonito@astropa.unipa.it

This is an Open Access article distributed under the terms of the Creative Commons Attribution License 2.0, which permits unrestricted use, distribution, and reproduction in any medium, provided the original work is properly cited. 
can generate shocks with temperature of a few millions degrees on the stellar surface which emit in soft X-rays (e.g. [1]).

The above picture is supported by time-dependent hydrodynamic and MHD models of radiative accretion shocks (e.g. [2-5]). All these models describe the impact of an accretion stream onto the stellar surface and show that the impact generates a reverse shock, which propagates through the accretion column, producing a hot slab. Although these models have allowed to investigate the dynamics and energetics of the shocked plasma, they did not provide a detailed study of the observability of the shocked plasma in the X-ray band, taking into account the effect of the dense and cold material surrounding the shocked column.

In fact, several open issues are still debated in the literature. For instance, to mention a few, the mass accretion rates derived from X-rays are, in general, sensibly lower (even by few orders of magnitude) than the corresponding mass accretion rates derived from UV/optical/NIR observations [6]. Also, the spectral analysis of X-ray emission arising from the impact region of accretion streams show that the shocked plasma seems to be denser at higher temperatures, a result which has been considered in contrast with the accretion shock models. In fact, several scenarios have been proposed to interpret the observations, invoking the interaction between the accretion stream and the corona [7].

The above issues prompted us to investigate, through an MHD model, the observability of accretion shocks in the X-ray band, taking into account the effects of local absorption, and providing useful diagnostics for a detailed and accurate interpretation of the observations. To this end, we use the 2D MHD model described by [8]. From the model results, we synthesize the X-ray emission arising from the impact region, taking into account the local absorption by the thick surrounding medium and the Doppler shift of lines due to the component of plasma velocity along the line-of-sight. In particular we focus on the line fluxes which traditionally are used for the density diagnostic in the observed spectra, namely the triplets of the He-like O VII $(21.6,21.8,22.1 \AA)$ and Ne IX $(13.45,13.55,13.7 \AA)$ lines. Finally, we compare the model results with the observations.

\section{The models}

We performed 2D MHD numerical simulations of an accretion stream impacting on the stellar surface of a CTTS (see [8] for more details). We assumed a fully ionized fluid with a ratio of specific heats $\gamma=5 / 3$ and treated as an ideal MHD plasma. The stream is modeled by numerically solving the time-dependent MHD equations of mass, momentum, and energy conservation, adopting cylindrical coordinates in the plane ( $\mathrm{r}, \mathrm{z}$ ) and assuming axisymmetry. The model takes into account all the relevant effect, namely the stellar magnetic field, the radiative losses from optically thin plasma, the thermal conduction (including the effects of heat flux saturation), the gravity, and a realistic description of the stellar atmosphere. The model is implemented using PLUTO ([9]), a modular, Godunov-type code for astrophysical plasmas.

The initial conditions represent an accretion stream with constant plasma density and velocity, propagating along the $\mathrm{z}$ axis through the stellar corona. The values of density and velocity of the stream are derived from the analysis of X-ray spectra of MP Mus [10]. We explore different configurations and strengths of the stellar magnetic field. in particular, we consider a magnetic field characterized by substantial tapering close to the chromosphere as expected by analogy with magnetic loop structures.

In all the simulations, the $2 \mathrm{D}$ cylindrical $(\mathrm{r}, \mathrm{z})$ mesh extends between 0 and $1.2 \times 10^{10} \mathrm{~cm}$ in the $r$ direction and between $-1.4 \times 10^{9} \mathrm{~cm}$ and $2.26 \times 10^{10} \mathrm{~cm}$ in the $z$ direction. The transition region between the chromosphere and the corona is initially located at $z=0$. The radial coordinate $r$ has been discretized uniformly with $N_{r}=512$ points, giving a resolution of $\Delta r \approx 2.3 \times 10^{7} \mathrm{~cm}$. The 


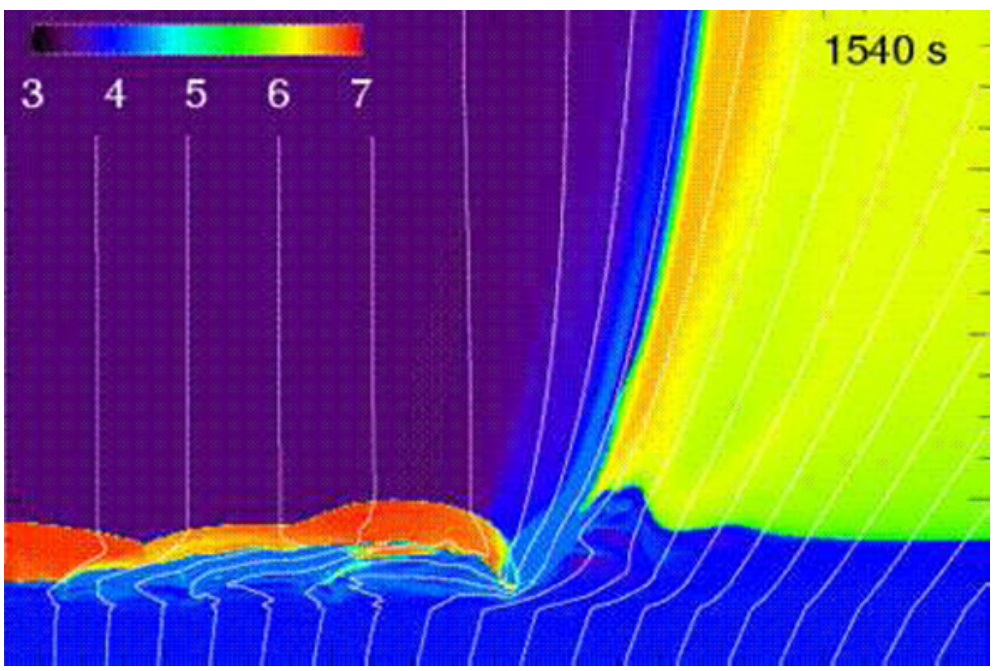

Figure 1. Temperature distribution of the model with $B=50 \mathrm{G}$ and a small tapering of the magnetic field lines

coordinate $z$ has been discretized on a nonuniform grid, giving a higher spatial resolution closer to the stellar chromosphere. A detailed description of the numerical setup can be found in [8].

From the results of the 2D simulations, we reconstruct the 3D spatial distributions of temperature and density, and the 3D velocity field. Taking into account the proper absorption coefficient [11], we synthesized the X-ray emission to be compared with the observations. From the synthetic maps and spectra of X-ray emission, we explore: 1) the effects of the local absorption (i.e. due to the chromosphere and the accretion stream itself) on the observability of the X-ray emitting plasma, for different line-of-sight (LOS) at which the impact region is observed; 2) the Doppler shift of detected lines.

\section{Results}

From the models described in [8], we synthesize the X-ray emission originating from the shocked plasma and derive maps and spectra assuming that the angle between the LoS and the stream axis is either $5 \mathrm{deg}$ or $45 \mathrm{deg}$. We explore the effects of the local absorption and the Doppler shift on the observability of the emitting plasma.

As an example, we show here the model with a magnetic field $B=50 \mathrm{G}$ and a small tapering of the magnetic field lines. Its temperature distribution is shown in Fig. 1. The left and the central panels of Fig. 2 show the X-ray maps for the 5 deg inclination case either with (central panel) or without (left panel) the effects of local absorption and Doppler shift. By comparing these two maps, we infer their influence on the emission: the part of the accretion shock rooted in the chromosphere is totally absorbed when the local absorption is taken into account. Therefore, the local absorption is crucial to determine the detectable emission rising from the accretion shock and cannot be neglected when comparing the model results with observations. The right panel of Fig. 2 shows the X-ray maps for the $45 \mathrm{deg}$ inclination case, taking into account the effects of local absorption and Doppler shift. By comparing the central and the right panels, therefore, we explore how the emerging X-ray emission depends on the LoS. We find that, for different LoS, different parts of the shocked slab contribute to the emerging X-ray emission: shocked plasma deeply rooted in the chromosphere suffers strong 


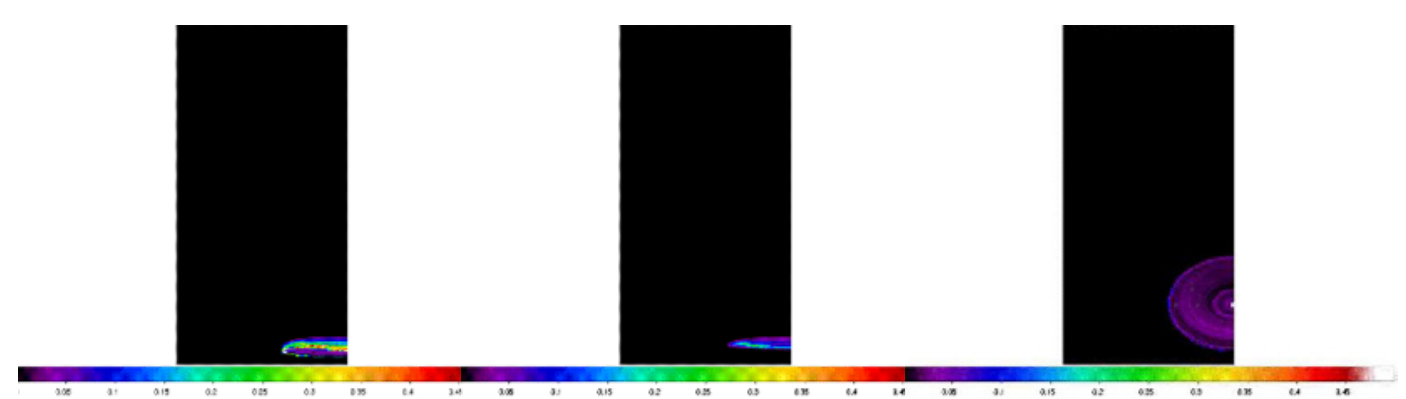

Figure 2. X-ray emission synthesized from our numerical models for the case of: 5 deg inclination without local absorption nor Doppler shift taken into account (left panel), 5 deg inclination (central panel), and 45 deg inclination (right panel) with all the effects taken into account

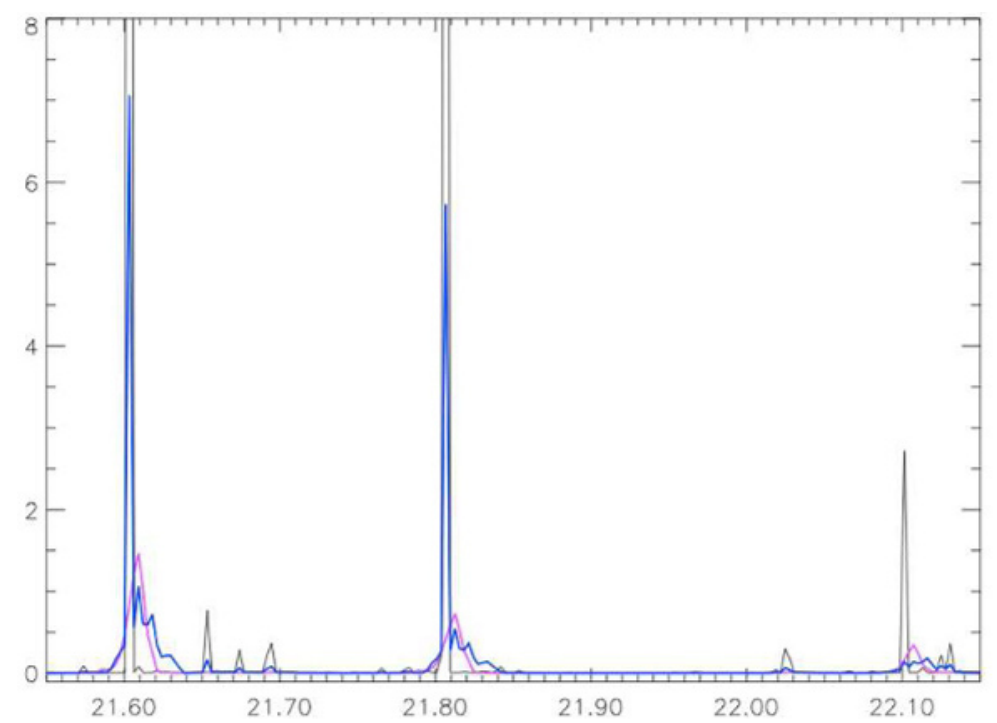

Figure 3. Enlargement of the spectra near the O VII triplet for the cases of: 5 deg without local absorption nor Doppler shift considered (in black), 5 deg (in magenta), and 45 deg (in blue) with all the effects taken into account

absorption if the LoS is at $5 \mathrm{deg}$, whereas it may contribute significantly to the X-ray emission at higher inclinations (45 deg).

We also find that the local absorption and the Doppler shift strongly influence the X-ray spectrum arising from the shocked plasma. Again, the effects of absorption and Doppler shift depend on the LoS at which the impact region is observed. Fig. 3 shows the influence of the local absorption and Doppler shift on the O VII triplet: when the absorption is taken into account (magenta line for the 5 deg case and blue line for the $45 \mathrm{deg}$ case), the flux of the lines are strongly reduced. Also, at $45 \mathrm{deg}$ (blue line in Fig. 3) the fluxes are higher than at 5 deg. In fact, as discussed above, at 45 deg shocked plasma rooted in the chromosphere (which is not detectable at $5 \mathrm{deg}$ ) contributes to the emerging $\mathrm{X}$-ray emission. 
Using the $f / i$ flux ratio derived for other He-like lines other than O VII (for instance Ne IX), it is possible to compare our model results directly with the observations to investigate whether the model can explain the evidence that the shocked plasma is denser at higher temperatures. Preliminary results seems to confirm this result.

\section{Discussions and conclusion}

We investigate the effects of local absorption and Doppler shift on the X-ray emission originating from the shocked plasma at the impact region of accretion streams falling on a CTTS. To this end, we consider a 2D MHD model of accretion shock, exploring different configurations and strengths of the magnetic field. From the model results, we synthesize the X-ray emission of the accretion shock formed at the interaction with the stellar surface, taking into account the effects of the local absorption and the Doppler shift. We find that the local absorption play a crucial role in determining the emerging $\mathrm{X}$-ray emission. We also find that the role of absorption change for different LoS. The comparison of model results with available observations is very promising and can shade light in the interpretation of the current open issues: why accretion rates derived in the X-ray band are lower than those derived in other wavelength bands; why the density of the shocked plasma inferred at higher temperature is higher.

\section{References}

[1] N. Calvet, E. Gullbring, ApJ 509, 802 (1998) apj

[2] A.V. Koldoba, G.V. Ustyugova, M.M. Romanova, R.V.E. Lovelace, MNRAS 388, 357 (2008), 0803.2020

[3] G.G. Sacco, C. Argiroffi, S. Orlando, A. Maggio, G. Peres, F. Reale, A\&A 491, L17 (2008), 0810.0192

[4] S. Orlando, G.G. Sacco, C. Argiroffi, F. Reale, G. Peres, A. Maggio, A\&A 510, A71 (2010), 0912. 1799

[5] T. Matsakos, J.P. Chièze, C. Stehlé, M. González, L. Ibgui, L. de Sá, T. Lanz, S. Orlando, R. Bonito, C. Argiroffi et al., ArXiv e-prints 557, A69 (2013), 1307. 5389

[6] R.L. Curran, C. Argiroffi, G.G. Sacco, S. Orlando, G. Peres, F. Reale, A. Maggio, A\&A 526, A104 (2011), 1011.5915

[7] N.S. Brickhouse, S.R. Cranmer, A.K. Dupree, G.J.M. Luna, S. Wolk, ApJ 710, 1835 (2010), 1001.0750

[8] S. Orlando, R. Bonito, C. Argiroffi, F. Reale, G. Peres, M. Miceli, et al. submitted, A\&A (2013)

[9] A. Mignone, G. Bodo, S. Massaglia, T. Matsakos, O. Tesileanu, C. Zanni, A. Ferrari, ApJS 170, 228 (2007), arXiv: astro-ph/0701854

[10] C. Argiroffi, A. Maggio, G. Peres, A\&A 465, L5 (2007), arXiv: astro-ph/0701765

[11] M. Balucinska-Church, D. McCammon, ApJ 400, 699 (1992) 